\title{
Online Disclosure of Incriminating Information: Patterns of Risky Information Behavior in Two Drug Forums
}

\author{
Kaitlin L. Costelloㄹ, John D. Martin III2, Ashlee Edwards ${ }^{2}$ \\ ${ }^{1}$ Rutgers, the State University of New Jersey \\ School of Communication and Information \\ Department of Library and Information Science \\ 4 Huntington Street \\ New Brunswick, NJ 08901 \\ k.costello@rutgers.edu \\ 2 University of North Carolina at Chapel Hill \\ School of Library and Information Science \\ 216 Lenoir Drive \\ Chapel Hill, NC 27599 \\ me@johndmart.in, aedwards@unc.edu
}

Author note: Correspondence concerning this article should be addressed to Kaitlin L. Costello. E-mail: k.costello@rutgers.edu

This is a preprint of an article accepted for publication in Journal of the Association for Information Science and Technology copyright (C) 2017 Association for Information Science and Technology (https://dx.doi.org/10.1002/asi.23880). 


\begin{abstract}
Although people disclose illicit activities such as drug use online, we currently know little about what information people choose to disclose and share or whether there are differences in behavior depending on the illicit activity being disclosed. This exploratory mixed-methods study examines how people discuss and disclose the use of two different drugs-marijuana and heroin-in the popular online forum Reddit. In this study hermeneutic content analysis is employed in order to describe the type of comments people make in forums dedicated to discussions about illicit drugs. With inductive analysis, seven categories of comments were identified: disclosure, instruction and advice, culture, community norms, moralizing, legality, and banter. Our subsequent quantitative analysis indicates that although the amounts of disclosure are similar in each subreddit, there are more instances of instruction and advice in discussions about opiates, and more examples of banter in comments about marijuana use. In fact, both subreddits have high rates of banter. We argue that banter fosters disclosure in both subreddits, and that banter and disclosure are linked with information seeking behaviors in online forums. This work has implications for future explorations of disclosure online and for public health interventions aimed at disseminating credible information about drug use to atrisk individuals.
\end{abstract}




\section{Introduction}

Although people disclose and discuss drug use on the open Internet, we currently do not know much about what information people choose to disclose or share; nor do we know if there are differences in information behaviors that are related to the specific illicit drug being discussed. Internet forums allow for a naturalistic examination of information behaviors in this context. Understanding the information behaviors surrounding illicit drug use will allow for the development of public health interventions that aim to successfully disseminate credible, justin-time information to drug users. This study also aims to better explicate the interplay between information seeking and personal information disclosure online, a growing area of interest in information science.

\section{Literature review}

Illicit drug use is prevalent in the United States: the National Institute on Drug Abuse estimates that over half (50.8\%) of all US adults over the age of 26 and $57.9 \%$ of US adults between the ages of 18 and 25 have used illicit drugs during their lifetime. Moreover, $2.7 \%$ of individuals over the age of 12 (7.1 million people) identify as dependent on illicit drugs within the last year; this percentage is higher (6.6\%) for adults between the ages of 18 and 25 (Substance Abuse and Mental Health Services Administration, 2015). However, while illicit drug use and abuse is not uncommon, we do not understand much about how people get information about illicit drugs. Mass media campaigns are one such source of information that have been well-studied (Wakefield, Loken, \& Hornik, 2010), but these campaigns deliver information to people and do not address active information seeking by individuals. In fact, we currently understand little about the information behaviors of people actively seeking out information about illicit drugs. Internet forums provide one such arena, wherein the information behaviors of drug users can be observed naturalistically. Online forums therefore provide a window into health the information behaviors surrounding drug use that are otherwise difficult to research given their stigmatized nature. Understanding these behaviors will inform future public health interventions aimed at either promoting safe drug use, or at decreasing the activity altogether.

There is a nascent body of research that examines online discussions of drug use on forums dedicated to the topic. The current literature on this topic focuses on two distinct areas: studies on stigma and anonymity (Barratt, 2011; Bowles \& Moretti, 2016; File \& Bahney, 2012) and studies on the impact of online discussions on public policy (Bancroft \& Reid, 2015; Barratt, Lenton, Maddox, \& Allen, 2016; Månsson \& Ekendahl, 2013; O’Brien, Chatwin, Jenkins, \& Measham, 2015; Zheluk, Quinn, \& Meylakhs, 2014). Most of the work in this area has been conducted in online forums containing discussions of psychoactive substances or "party drugs" such as psilocybin mushrooms, LSD, Ketamine (Tackett-Gibson, 2008), or Ecstasy (Murguia, Tackett-Gibson, \& Lessem, 2007). A smaller body of work looks at marijuana use (File \& Bahney, 2012; Månsson \& Ekendahl, 2013), and very few studies address online discussions of opioids such as heroin or prescription painkiller medications. As O'Brien et al. note, "There remains a lack of relevant social research to draw upon [in this area]. Only a handful of responses have moved beyond prevalence to explore user experiences and motivations" (2015). Our study aims to address this gap by examining the content of online discussions with a specific focus on information behavior in order to uncover user experiences and motivations as they seek, disclose, or exchange information about drugs online. 
We focus on information behaviors in this context for several reasons. Most of the models of information behavior conceive of information needs, seeking, and use as separate and distinct processes (Case, 2012). However, a growing body of literature demonstrates that information needs, seeking, and use are not separate, distinct activities but are closely interwoven processes that often occur simultaneously and concurrently (Costello, 2015). While this has been examined in the context of non-stigmatized chronic illness, it has not yet been researched in other contexts. The present study therefore aims to further examine the interplay of information seeking and information disclosure. Like some health conditions, drug use is often stigmatized and kept private (Ahern, Stuber, \& Galea, 2007). It is therefore an ideal context for studying disclosure behaviors, as disclosure operates in a dialectic with privacy (Petronio, 2002). Furthermore, the ability to be anonymous on the Internet makes disclosure of sensitive content like drug use more likely (Peddinti, Ross, \& Cappos, 2014), as anonymity frees people from the fear of judgment (Pedersen, 1997). Finally, our understanding of information behavior as it relates to drug use is limited to offline interactions (Becker, 1953; Thomas, Dunn, Swift, \& Burns, 2011; Todd, 1999). However, people are increasingly engaging in information exchange online. This study therefore fills gaps in our understanding of the interaction between information seeking and personal information disclosure in online environments and has implications for public health interventions that target these information behaviors in the context of illicit drug use.

\section{Methods}

The overarching question motivating this mixed-methods exploratory study is: How do people discuss and disclose incriminating or illicit drug behaviors online? To address this question, we use hermeneutic content analysis. Hermeneutic content analysis begins with an initial qualitative content analysis of a test set of text; this is followed by a qualitative and quantitative dimensional analysis of a new sample of textual data in order to identify patterns among and between the codes developed in the initial qualitative content analysis (Bergman, 2010).

We choose to examine subforums, or subreddits, on Reddit, because of its popularity. It it is the $7^{\text {th }}$ most popular website in the United States and it has over 200 million active and unique users (cite Alexa here?). Reddit bills itself as "the front page of the Internet," and is a community-driven, corporate-owned discussion board comprising sub-forums-called "subreddits"—on myriad topics and subjects. Subreddits are established, maintained and moderated entirely by users, who also create content by starting threads and posting comments in existing threads. Individual thread topics often extend into the realm of the legally questionable; indeed, there are entire subreddits devoted to specific illegal behaviors. Although Reddit has recently begun to remove forums that seem to victimize specific groups, partially in response to increased scrutiny on the site owners and administrators as enablers of criminal activity (Isaac, 2014), Reddit does allow users to discuss illicit and illegal activities.

In this study, we began with a thematic analysis of 800 comments posted to two different subreddits where discussions of illicit activity were common: $r$ /trees, a marijuana forum; and $r$ /opiates, a forum to discuss opioid use. To choose these three subreddits, we performed an initial survey of subreddits where disclosure of many types of criminal behavior were frequently discussed, including but not limited to drug use, media piracy, theft, domestic assault, and violence. We excluded private subreddits and those that were inactive, and then developed 
our initial qualitative thematic analysis based on the two most active and heavily populated subreddits, which both focus on illicit drug use.

Our thematic analysis began with inductive qualitative content analysis; our codebook was developed iteratively and the codes it contained were progressively refined and categorized during eight rounds of individual coding and eight subsequent peer-debriefing sessions (Lincoln \& Guba, 1975). In peer debriefing, we discussed the codes as they were being developed and collectively identified 21 major themes in our analysis. These seven themes were grouped into seven interrelated categories (Saldana, 2015). Our final codebook contains 21 codes organized into eight categories, as illustrated in Table 1 . The codes and categories are mutually exclusive and exhaustive. During the coding process, we also gathered contextual information about comments when necessary. For example, we followed links in comments to aid us in properly understanding outside references and compiled a glossary of vernacular specific to our chosen forums.

Table 1.

Codebook with categories, codes, descriptions, and examples.

\begin{tabular}{|c|c|c|c|}
\hline Category & Codes & Description & Examples \\
\hline \multirow[t]{3}{*}{ Disclosure } & DIS & Disclosing an illicit act & $\begin{array}{l}\text { "I'm } 19 \text {, smoke } 1 \text { gram a day, } \\
\text { and work at a call center." }\end{array}$ \\
\hline & CXT & $\begin{array}{l}\text { Providing contextual } \\
\text { information for an illicit } \\
\text { disclosure }\end{array}$ & $\begin{array}{l}\text { "I'm not sure. They are small } \\
\text { pills and he only gave me 5." }\end{array}$ \\
\hline & ASK & $\begin{array}{l}\text { Asking about the illicit } \\
\text { activity }\end{array}$ & $\begin{array}{l}\text { "I'm ignorant about street prices. } \\
\text { How much should a } 5 \mathrm{mg} \text { vicodin } \\
\text { cost me?" }\end{array}$ \\
\hline \multirow[t]{3}{*}{ Instruction } & INS & Instructing or giving advice & $\begin{array}{l}\text { "One final tip, make sure you } \\
\text { stay hydrated when you're } \\
\text { smoking and don't overeat! That } \\
\text { will give you headaches } \\
\text { tomorrow. Happy smoking!” }\end{array}$ \\
\hline & $\mathrm{OTH}$ & $\begin{array}{l}\text { Referring to other } \\
\text { forums/platforms/links }\end{array}$ & $\begin{array}{l}\text { "Don't be afraid! Check out this } \\
\text { guy's youtube channel, he } \\
\text { describes how to get high safely } \\
\text { really well." }\end{array}$ \\
\hline & WAY & $\begin{array}{l}\text { Ways to disclose illicit } \\
\text { activities offline }\end{array}$ & $\begin{array}{l}\text { "You can tell her a story to try to } \\
\text { bring up the subject but you } \\
\text { might not like what you hear." }\end{array}$ \\
\hline \multirow[t]{2}{*}{ Drug culture } & CUL & References to drug culture & $\begin{array}{l}\text { "I know where this myth got } \\
\text { started, it comes from the pro- } \\
\text { cannabis movement." }\end{array}$ \\
\hline & COD & Using coded language & $\begin{array}{l}\text { "I used to call marijuana 'pizza.' } \\
\text { A small amount was a slice and a } \\
\text { lot was a pie." }\end{array}$ \\
\hline Community norms & $\mathrm{COM}$ & $\begin{array}{l}\text { Referring to community } \\
\text { rules/norms }\end{array}$ & $\begin{array}{l}\text { "It confuses me to see people on } \\
\text { other drug forums using actual } \\
\text { photos of themselves for profile } \\
\text { pics. We don't do that here. Why } \\
\text { would you take that chance?" }\end{array}$ \\
\hline
\end{tabular}




\begin{tabular}{|c|c|c|c|}
\hline & RUL & Emphasizing subreddit rules & $\begin{array}{l}\text { "To prevent trolling, we don't } \\
\text { allow comments from users with } \\
\text { negative karma on Reddit here." }\end{array}$ \\
\hline \multirow[t]{5}{*}{ Moralizing } & MOR & Moralizing statements & $\begin{array}{l}\text { "Please educate yourself before } \\
\text { doing drugs." }\end{array}$ \\
\hline & ADM & $\begin{array}{l}\text { Admonishment of } \\
\text { user/activity }\end{array}$ & $\begin{array}{l}\text { "You should feel bad about this. } \\
\text { Meetings aren't my thing, but if } \\
\text { you're going to go, don't get high } \\
\text { in the bathroom while you're } \\
\text { there! It's disrespectful to the } \\
\text { people trying to get clean." }\end{array}$ \\
\hline & GUI & $\begin{array}{l}\text { Contrition, guilt, self-loathing } \\
\text { over act }\end{array}$ & $\begin{array}{l}\text { "I am a sad and miserable waste } \\
\text { of life right now, staring at my } \\
\text { phone waiting for my dealer to } \\
\text { text me back. If I had a nickel for } \\
\text { every hour I have wasted } \\
\text { waiting around for drugs..." }\end{array}$ \\
\hline & CND & $\begin{array}{l}\text { Condoning or praising the } \\
\text { illicit activity }\end{array}$ & $\begin{array}{l}\text { "I'm happy to hear a story like } \\
\text { this. I went through something } \\
\text { similar, and truly believe } \\
\text { controlled and proper drug use } \\
\text { can change lives for the better." }\end{array}$ \\
\hline & JST & Justifying the illicit activity & $\begin{array}{l}\text { "I've seen way worse incidents } \\
\text { happen, people being losers and } \\
\text { idiots, from drinking and not } \\
\text { smoking." }\end{array}$ \\
\hline Legality & LEG & $\begin{array}{l}\text { Suggesting legal alternatives } \\
\text { to illicit activity }\end{array}$ & $\begin{array}{l}\text { "Have you ever considered } \\
\text { suboxone, methadone, or } \\
\text { kratom? Many people who quit } \\
\text { opiates have found them useful." }\end{array}$ \\
\hline \multirow[t]{4}{*}{ Banter } & BNT & $\begin{array}{l}\text { Banter, tangent, derail, } \\
\text { unrelated topic }\end{array}$ & $\begin{array}{l}\text { "I'm allergic to dogs, but my cat } \\
\text { feels like a velvet baby. I love my } \\
\text { cats." }\end{array}$ \\
\hline & GRA & $\begin{array}{l}\text { Gratitude for advice or } \\
\text { information }\end{array}$ & $\begin{array}{l}\text { "Thanks! You guys are the best } \\
\text { and this is why I love r/trees. } \\
\text { You guys get it." }\end{array}$ \\
\hline & REA & $\begin{array}{l}\text { Reassuring over fears related } \\
\text { to illicit activity }\end{array}$ & $\begin{array}{l}\text { "It's ok. This happens. Chalk it up } \\
\text { to experience." }\end{array}$ \\
\hline & SOC & $\begin{array}{l}\text { Socially supportive } \\
\text { statements }\end{array}$ & $\begin{array}{l}\text { "I don't have much advice, but I } \\
\text { do have kind words. I'm sorry } \\
\text { this happened. I know it's hard. } \\
\text { Private message me if you ever } \\
\text { need to talk." }\end{array}$ \\
\hline
\end{tabular}

In the subsequent quantitative phase of our hermeneutic content analysis, we used webscraping techniques over the course of five days in August 2014 to gather all front-page posts and their associated comments from each subreddit. All top-level threads and replies on each were collected, allowing enough time to elapse so that the thread activity had peaked (i.e., we scraped threads three days after they had been initially posted). This technique allowed us to minimize topical bias within the samples as they came from the same period of time (Norris, 
1997) In our analysis, we do not differentiate between posts and comments. We then coded each of the posts and comments and met to discuss all disagreements; this process is called interpretive convergence and relies on intensive group discussions leading to consensus (Saldana, 2015). We then conducted a quantitative comparison using Pearson's chi-square test between the subreddits for each category of analysis to analyze differences between the subreddits (Agresti \& Kateri, 2011; Bergman, 2010).

Throughout our results section, quotes have been edited for grammar, and inconsequential details have been edited so that deductive disclosure of the individuals who have made comments that appear in this article is less likely. This decision was made to protect the individuals discussing illicit behavior; direct quotations used in this paper for research purposes are available upon request.

\section{Results}

Descriptive information about our dataset, including the number of unique threads, comments, and users, is available in Table 2.

Table 2.

Descriptive information about sample

\begin{tabular}{rrrr} 
& Threads & Comments & Unique users \\
\hline r/opiates & 62 & 1,266 & 355 \\
r/trees & 57 & 1,305 & 875 \\
Total & 121 & 2,571 & $1225^{*}$
\end{tabular}

*There are 5 users who made comments in both or/opiates and $r /$ trees.

Both implicit and explicit admissions of drug use were identified in comments on both subreddits. There are 355 unique users commenting in $\mathrm{r}$ /opiates with 1,266 total comments in 62 threads. The distribution of codes for $r$ /opiates is shown in Figure 1. There are 875 unique commenters in our sample of $\mathrm{r}$ /trees with 1,305 comments in 57 threads. The distribution of codes for $r$ /trees is shown in Figure 2. 


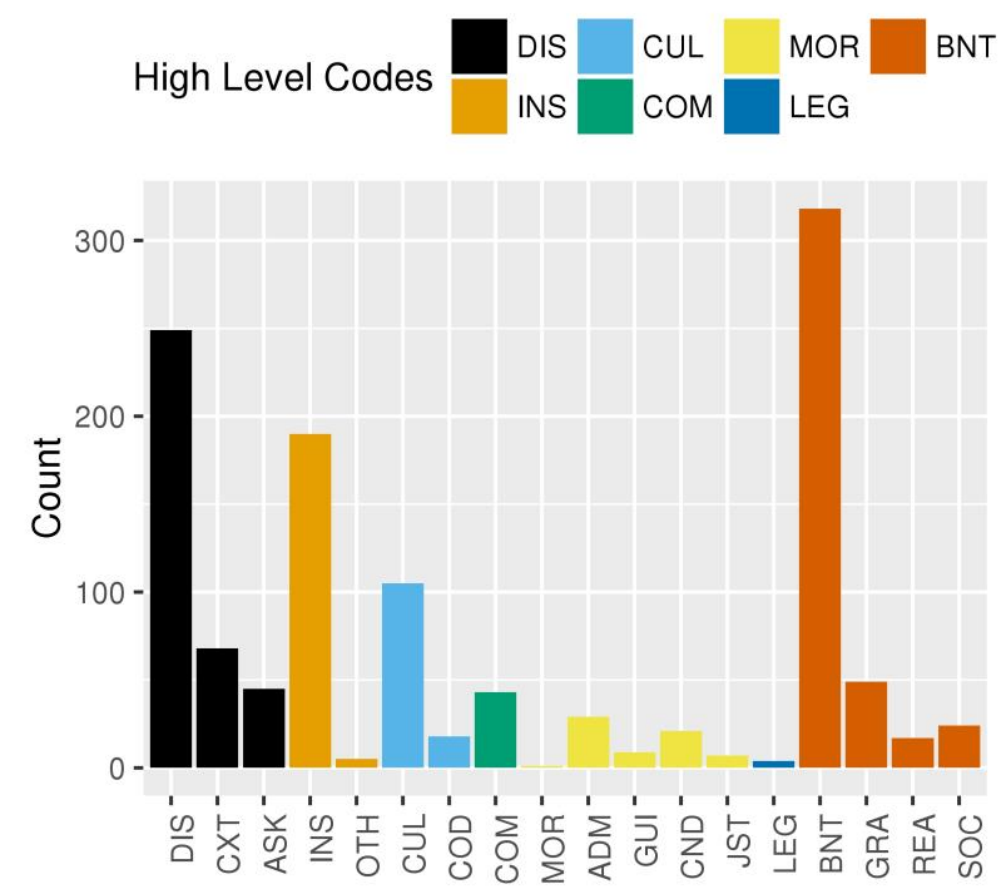

Figure 1: All codes in r/opiates

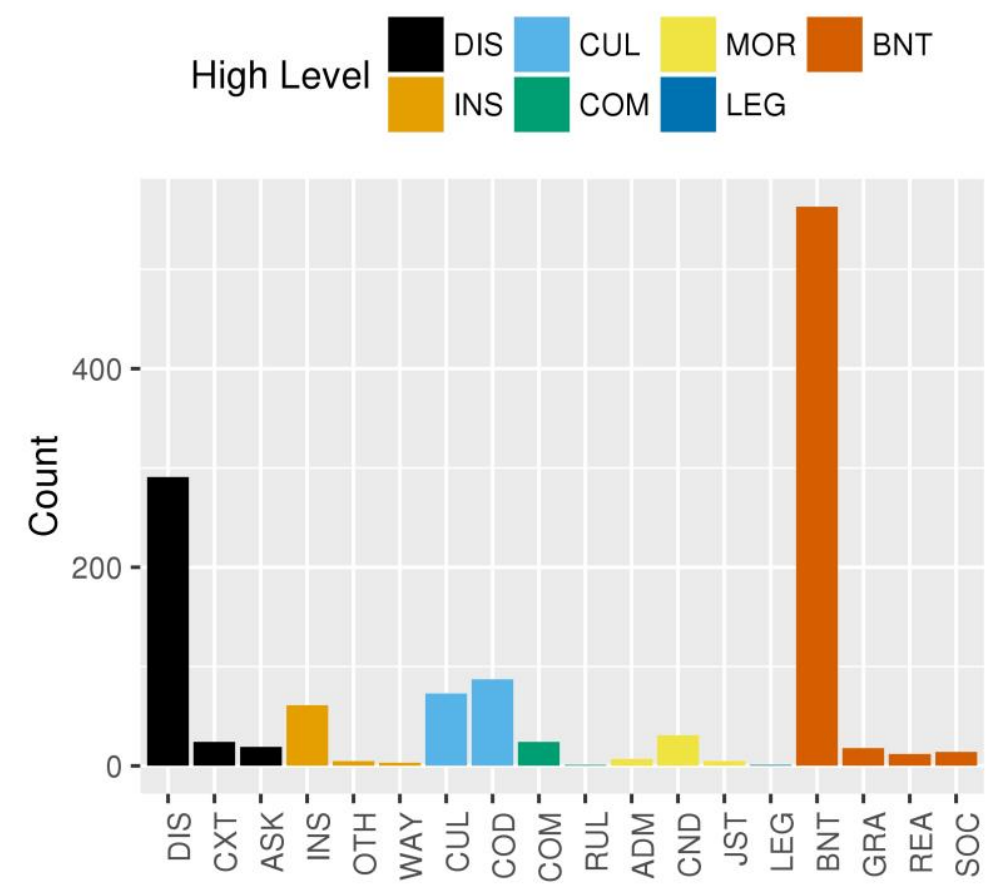

Figure 2: All codes in $\mathrm{r} /$ trees

\section{Banter}

Banter is prevalent on both subreddits and is the most common type of comment. Rather than only using the forums to seek out or share information about using drugs, commenters on both subreddits tend to make off-topic comments. Most of the banter in both subreddits consists of jokes and witty repartee. In many cases, one joke will set off a series of related jokey comments. For example, one long discussion in $\mathrm{r}$ /trees ensues about spider webs and their strength; most 
of the comments in this discussion are short, one-liners that are posted in rapid succession. From these discussions, it is clear that banter begets banter in both subreddits.

There are multiple types of banter that are not just jokes: people talk about hobbies, like watching television, playing video games, and writing. They also discuss their jobs, where they live, what they like to eat, and dating. In $\mathrm{r} /$ trees, there are multiple threads where people share song titles and YouTube links for music, often with the stated goal of aiding others in creating playlists to listen to while using drugs. These comments illustrate that these subreddits are communities for some users; in fact, there are many expressions of social support, reassurance, and gratitude embedded in some of the banter on both subreddits: "Thank you to everyone who responded to me with advice, kind words, and their experiences. I appreciate it more than you guys probably know. Knowing that I'm not alone helps." In some cases, people discuss the role that the subreddit plays in their daily lives:

$\mathrm{Hi}, \mathrm{r} /$ opiates. I want to say that you guys are awesome. If anyone ever feels like nobody cares about them, remember that I care. I know this is kind of off topic, but I also know that many of us have depression (me included) and it is always good to know there's someone to talk to. I hope you all stay safe.

Interestingly, these expressions of social support often note their off-topic nature.

\section{Disclosure}

In both subreddits, people have three main reasons for disclosing illicit drug use: they do so to give advice to others, to get information from others, or to provide context for illicit disclosures they made previously in the thread. Soliciting information takes multiple forms. In r/opiates, users ask about handling addiction and for information about actually administering the drug in the immediate future. Addiction is a common topic in disclosive comments on r/opiates: "I don't know if a life without heroin will ever happen for me, because of lack of willpower and because I truly love it. However, I do a lot less now." These threads often combine socially supportive comments with disclosure, and these two codes are often intertwined throughout comments in both subreddits. Some users disclose their own struggles with addiction and offer social support in the same comment, while other users simply offer social support without disclosing personal information. The strong sense of identification within these disclosive comments about addiction is striking: "Hello, past me," says one commenter in response to a long thread about addiction. In multiple instances, these discussions concern attempts to quit using opiates by replacing them with other illicit substances: "I swear by edible marijuana. I am Paula Weed when detoxing. It kills the pain and legs shaking, it lets me eat and keeps me from puking, and it keeps me from going kind of nuts from detox." Coping with addiction is a frequent topic in this subreddit, and it is one that tends to involve a fair amount of disclosure. For example, some people who ask for help with their addiction only want to take a "break," and commenters respond in kind: "I had to hit my own rock bottom before anything changed, but I still use two times a month. If you can do this, I recommend it, but only you know the limits of your selfcontrol." In r/trees, this is called a "t-break," or tolerance break, and is used as a technique to "reset" one's tolerance level to marijuana.

On $\mathrm{r}$ /trees, addiction to marijuana is not a frequent topic of conversation, but people do disclose their use when they talk about how much they ingest daily. These disclosures are often in response to prompts from others; e.g., "What does it feel like when you're 'too high?"” Disclosure of substance use on both subreddits builds a sense of camaraderie in the group. In fact, users on 
$\mathrm{r} /$ trees have developed a shorthand, "insider" method for disclosing their current level of intoxication. On $\mathrm{r}$ /trees, people include a number between one and ten in brackets for this purpose: “I'm so high. Thanks for being here, $r /$ trees [9]". Because of this feature, many comments that might have otherwise been coded as banter on this subreddit were coded as disclosive statements; e.g., "That made me laugh out loud [6]." In fact, disclosure on this subreddit often happens in threads where people are sharing stories about their lives and discussing topics tangential to marijuana use.

Disclosure also functions as a point of commiseration in r/opiates. Posters and commenters discuss their opioid of choice, often immediately after ingesting it, and invite others to talk about it with them: "Just woke up, popped a blue, snorted another, and I still have one left. Now I'm just hanging out. Hope everyone has a great day!" Other commenters then join the thread; some of them are currently high, while others simply celebrate the fact that the original poster has done drugs. In some cases, people also talk about other non-opioid drugs they are consuming, including ecstasy and marijuana: "I'm an upstanding member of society. I have a family, volunteer, and donate to charity. I also love opiates, alcohol, Valium, MDMA, Xanax, acid, ketamine, heroin, heroin, and heroin."

People on r/opiates also ask disclosive questions about how to use opioids safely. Sometimes, they qualify their choice to ask the question in this particular forum: "Sorry, I don't know much about the effects of opiates and couldn't think of way to put this feeling into words and search for it using Google." Users often apologize and note that they could search for information another way; in these comments, they say the forum is easy to use and offers just-in-time information that is trustworthy: "Yeah I know, '[you idiot] use Google' or 'just search through the sub,' but will lope help me with cravings?" asks one user.

People also ask about ingesting drugs in the present moment. For example, one user in r/opiates asks: "I want to eat an oxy right now and do that chewing thing. How long does it take to kick in?" Several individuals reply with disclosive information, including comments such as: "I find that it takes about 30 minutes" and "Chewing doesn't work, the time release makes them coagulate back together in your stomach. Trust me, I've thrown it up." Users also ask whether or not a given experience with opiates is "normal." For example, one individual had an unexpected reaction to heroin: "I have no idea why this happened, and I was curious if anyone else experienced this when coming down?" Other participants in the thread corroborate the experiences of the original poster, disclosing their own drug use in the process. Many of the questions about the logistics of drug administration are about the efficacy and potential lethality of combinations of multiple drugs; e.g., "I know benzodiazepines and opiates have strong synergistic effects. I want to get high, but I want to be safe too. What's the recommended combination?" Therefore, on this subreddit, disclosure and information seeking are often linked processes: users post personal information about themselves in order to get relevant advice from other commenters, or to lend credence to the advice they're giving.

Like in the r/opiates subreddit, many of the conversations about using marijuana discuss the challenges of maintaining a "normal" life while being a heavy drug user. One thread in particular that illustrates this well in $\mathrm{r} /$ trees is a long discussion about whether or not parents-of both young and older children - should smoke marijuana, and if they should disclose their drug use to their spouse: "My [wife] fully supported it, especially after I was no longer suffering from insomnia or back pain," says one user. Others give advice about how to balance parenting with marijuana use: "I never toke around the kids, and I keep it locked up. I only vaporize." Perhaps 
surprisingly, this thread contains very few moralizing statements; only one user replies with admonishment: "No! Do not do that!" Other conversations of this nature deal with balancing work, school, or other commitments with marijuana use.

Relatedly, many people in both subreddits talk about using illicit substances as a way to cope with other medical issues, such as chronic pain or anxiety. For example, one r/trees user explicitly asks for advice about managing other medical issues with marijuana:

I need to get some perspective. I am a daily smoker, but it's not just recreational. I experience nausea, have endometriosis, and have anxiety. I think I'm concerned because some friends think that I'm exaggerating my health as an excuse to overuse the drug. I do have to qualify that it is not legal where I am. Given this information, what do you guys think? Do I need to slow down, or am I good?

Multiple people respond to this question with disclosures of their own, both of their own medical conditions and about their own illicit drug use. This indicates that in some cases, disclosure prompts disclosure.

\section{Instruction}

In contrast, comments that provide recommendations based on anecdotal information or without citing personal experience are not disclosive, but instructional. Nearly all of these comments are unsourced; that is, the reader cannot tell how the commenter knows the information that they are sharing, because its source is not given. In a few cases, however, people link out to other resources online: either to other threads within the subreddit, or to other websites. Links out to other resources are nearly always devoid of any other text or contextual information. In instructive comments, individuals do not explicitly refer to their own experiences, thus avoiding direct disclosure of drug use by engaging in information sharing rather than personal information disclosure. In many cases, instructive comments directly follow disclosive comments: people will ask, while disclosing their illicit drug use, how to ingest a drug; the responses will not contain personally disclosive information, but instead contain unsourced advice.

In instructive comments, people frame their advice as factual; e.g., "you will get higher more quickly if you chew the pills," without actually saying how they know the information. These comments may also use scientific language; in the same thread, someone says the same thing, but with very different terminology: "The highest bioavailability and mechanism of action is sublingual." Use of scientific language was not seen in other types of comments, indicating that perhaps it is being employed increase a sense of credibility in cases where a source is not given.

In response to solicitations for advice, which may or may not be disclosive, commenters will often ask for additional contextual information, such as the tolerance of the original poster or the other drugs the poster has consumed in order to suss out potential interactions: "It depends on your personal physiology. Can you elaborate? I'll help if I can." These comments often contain warnings about the danger of using opiates with a low tolerance level. For example, in a response to a query about mixing opiates with an anti-anxiety medication, one commenter says: "This can be incredibly dangerous. But since you're going to do it anyhow...." This commenter then gives more detailed advice without disclosing information about themselves. In many cases, these instructive responses skirt the line of disclosure, nearly disclosing personal information without doing so; e.g., "I would definitely only do a little at first." Sometimes, people discuss how to pass drug tests while still ingesting illicit substances; again, these comments are 
instructive without being disclosive. Finally, some instructive comments contain advice on how to talk about using drugs with non-users: "Just tell people it isn't any of their business. Repeat it until they say no, and walk away."

\section{Drug culture}

Discussions about drug culture, or that refer to drug culture, are present in both subreddits. In these comments, people discuss purchasing drugs, dealing with law enforcement, and coded language. Threads about purchasing drugs often contain comments where users compare the prices of drugs in their location with one another. "Tar can be a way better deal. You can sometimes get it for $\$ 60-\$ 120$ a gram, while powder goes for $\$ 140-\$ 200$," says one person in a thread about purchasing different opioids from a dealer on the street. In some of these conversations, participants are often scornful of people-dealers or non-users-who do not seem to know the monetary value of drugs: "Wow. If I am paying that much, I want something stronger."

Many of the comments about interacting face-to-face with other drug users employ or define coded language and include questions and responses about what certain code words or phrases mean, and discussions about using code words in face-to-face environments as a way to identify or get the attention of other drug users. These comments do not merely employ coded language: they consist of commentary about the use of coded language itself. For example, users talk about the "secret codes" they use when discussing marijuana; these codes serve two purposes: they help them identify other smokers, and they mask the purpose of their conversation so that outsiders will not learn that they smoke marijuana. "I call it Fred. It's incredibly easy to slip a name into a conversation. 'Fred stopped by; he wants you to come chill.'" Comments about coded language are often short and to the point; they also are often quite witty, particularly when they are about using language offline to identify other people who use marijuana. Many of the comments about drug culture focus on offline interactions. In $\mathrm{r} /$ trees, these discussions are concerned with the popular conception of marijuana users as lazy, unemployed, and uneducated: "I had a conversation with a couple about marijuana, and my use came up. It had been both civil and speculative up until that point, but then they went on about how I might as well been a bum." These conversations serve to strengthen the bonds between users on this subreddit: "This is why I love r/trees," says one commenter in a lengthy discussion about how popular culture frames marijuana use: "You guys get it!"

In some comments about drug culture, people discuss the reasons they have for remaining circumspect about drug use: for example, in one case commenters debate the usefulness of posting about their own drug use using the acronym "SWIM"- "Someone Who Isn't Me." This tactic distances one's offline drug use from one's online persona. Other tactics for masking identities while disclosing incriminating information online are also discussed. For example, one commenter says that they don't provide any personal photographs on the site: "I just say 'I did a, b, or c,' but I don't use a picture of myself in my avatar online."

Finally, people have discussions about the role law enforcement plays in drug culture. Often people tell stories of nearly being caught by police. For example, one individual on r/opiates had a run-in with the police when he was high; he claims he had drugs in his car, but they were not discovered. Although he was not arrested nor was he charged with a crime, he recounts: "He said he would drop the charges if I would narc on people. I told him I wasn't trying to be killed... I just don't believe in narcing. We are all in this together on this side of the war on drugs." 


\section{Moralizing}

There are four types of moralizing statements made in both subreddits: admonishment and condoning are two forms of moralizing directed at other users, while guilt and justification are moralizing statements made about the self. Admonishments on r/opiates are often reprimands regarding unsafe drug practices: "Xanax on hand in case you take too much morphine? Stop right there." Like in many other comments, personal experience is highly valued and is often expressed in admonishments. Interestingly, several of the threads that contained high levels of admonishment dealt with the topic of mixing Xanax, a benzodiazepine, with opiates. In some cases, these admonishing statements also offer a good deal of factual information about why mixing these substances is not advisable. Commenters also urge people to learn more about the interaction by seeking out information on their own: "Lots of opiate deaths happen because they mixed them with Xanax. Promise me you'll research interactions and educate yourself."

In contrast, comments that condone the behavior of other users are often more supportive in tone; in most cases, they are responses to people who have quit using opiates but are still on the subreddit: "I'm proud of you. It's not easy, but you've been sober for five months. Keep it up." In contrast to r/opiates, where users condone people quitting drugs, users on $\mathrm{r} /$ trees tend to condone the illicit behaviors of other marijuana users. For example, in a discussion about how much marijuana use is appropriate on a daily basis, several people say things like "You do you. As long as it doesn't interfere with your normal daily life, you're not smoking too much."

People also justify their own marijuana use by saying things like "Being high makes you more curious, and it's made it so fun for me to learn about new things." However, some users express guilt over using illicit substances, particularly when there are negative consequences to their actions: "Got caught smoking in my room last night, and I'm in trouble at school. Sucks. It's entirely my fault and I know that. Learn from your mistakes, fellow friends." This also occurs in the opiates subreddit, where people express guilt about starting to use drugs in the first place and guilt over relapsing, although in some cases these expressions of guilt are a mixture of guilt and justification: "Went to rehab to show people I was serious about getting help, regain trust, and to learn about myself and fight my demons, and hopefully come out the other side a better man able to stay clean. In reality, it was shit, I relapsed the day I got out." This commenter then goes on to blame his relapse on the quality of care he received in the rehabilitation center, demonstrating that guilt and justification can occur concurrently in some cases.

\section{Community norms}

These comments are largely about how people communicate within each subreddit. People discuss the credibility_or lack thereof_of other users on the subreddit; for example, in a conversation about tolerance on $\mathrm{r}$ /opiates, multiple people get upset about what they consider to be dangerous misinformation that was shared: "Once again, someone here has the audacity to spew ignorant opinions, probably based on their one experience, as some kind of medical 'fact."' People also discuss the most common questions that are asked; in one case, a user distinguishes between "novice" and "expert" users and the type of questions they might ask: "There are a lot of frequently asked questions here. They're posted on the sidebar, but novice users ignore the FAQ and just post their question." Comments about community norms on $\mathrm{r} /$ trees are rare, but in some cases people discuss the use of upvotes, a convention on Reddit for "liking" a post, which they call "uptokes" in reference to marijuana slang. 
Although very few comments in the sample that address legal alternatives in either subreddit, the comments that do in $\mathrm{r} /$ opiates suggest replacing opiates with other legal substances such as suboxone, or quasi-legal alternatives such as kratom. This is also the case in the $\mathrm{r} /$ trees subreddit, where people recommend alcohol consumption as a legal alternative to marijuana use.

\section{Comparing r/trees with r/opiates}

From the qualitative examination of both subreddits, it is clear that there are similarities in the type and quality of discussions in both $\mathrm{r} /$ trees and $\mathrm{r}$ /opiates. Discussions contain a great deal of joking and banter, but people also disclose their own drug use, often in the course of information seeking activities. Disclosure of drug use provides valuable and often necessary context both when asking questions and when people answer questions posed by others. Although the subreddits are qualitatively similar, a Pearson's Chi-squared test of independence reveals statistically significant differences between the two subreddits $\left(\chi^{2}=117.94, \mathrm{df}=6\right.$, $p<4.41 e-23$ ). To determine which categories made the largest contribution to these observed differences, we computed standardized residuals for each category as seen in Table 3. This statistical test controls for the difference in size between both subreddits.

Table 3.

Chi-square observed, expected, and residual values

\begin{tabular}{llrrrrrrr} 
& $\chi^{2}$ & DIS & INS & CUL & COM & MOR & LEG & BNT \\
\hline r/trees & Expected & 390.49 & 131.47 & 142.31 & 35.45 & 55.64 & 2.95 & 507.68 \\
& Observed & 413.00 & 197.00 & 125.00 & 45.00 & 69.00 & 4.00 & 413.00 \\
& Std. Res. & 1.92 & 8.47 & -2.16 & 2.28 & 2.57 & 0.85 & -7.62 \\
r/opiates & Expected & 402.51 & 135.53 & 146.69 & 36.55 & 57.36 & 3.05 & 523.32 \\
& Observed & 380.00 & 70.00 & 164.00 & 27.00 & 44.00 & 2.00 & 618.00 \\
& & & & & & & & \\
& Std. Res. & -1.92 & -8.47 & 2.16 & -2.28 & -2.57 & -0.85 & 7.62
\end{tabular}

Two categories are contribute greatly to the significant difference between $\mathrm{r} /$ trees and r/opiates: instruction and banter. There is a more instruction than expected in $\mathrm{r} / \mathrm{opiates}$, and more banter than expected in $\mathrm{r} /$ trees. This can also be seen in the radial diagrams in Figures 3 and 4. Interestingly, although the users in $\mathrm{r}$ /trees have shorthand for disclosing their intoxication with marijuana while they are commenting, this does not have much an effect on the actual amount of disclosure in the subreddit. 


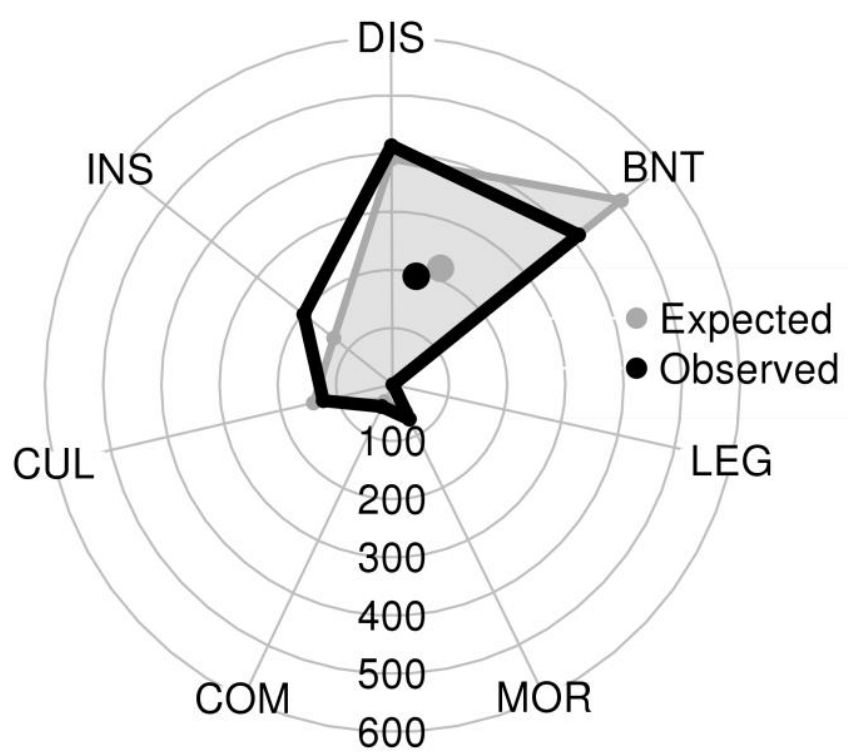

Figure 3. Difference in expected v. observed: /r/opiates

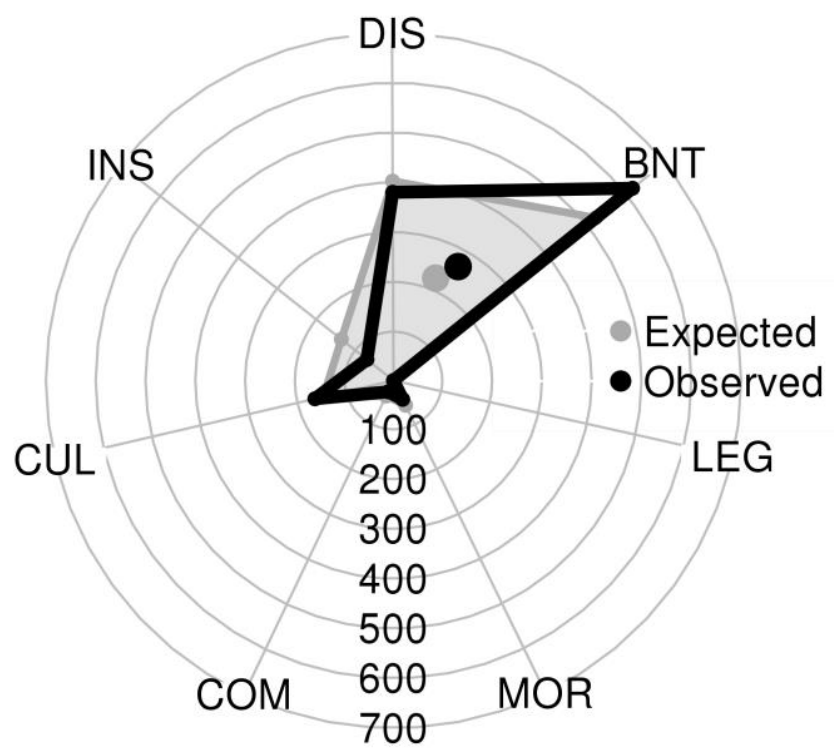

Figure 4. Difference in expected v. observed: /r/trees 


\section{Discussion}

An unsurprising but noteworthy finding in this study is the prevalence of banter in both subreddits-comments and discussions unrelated to drug use, which are the most common type of comment on both subreddits. Often, especially in $\mathrm{r} /$ trees, these comments are jokes. Although these comments seem to be non-sequiturs that serve little purpose, they are actually examples of phatic communication: messages that are intended not to convey information, but to build or maintain social relationships (Žegarac, 1998). One example of phatic communication is banter, jocular expressions that may be seen as offensive by individuals who do not "get" the joke (Plester \& Sayers, 2007). Indeed, much of the banter in our sample was offensive, as is common on Reddit, which has been called "a chaotic space that is at times both compelling and repulsive" (Massanari, 2015). Indeed, a recent survey indicates that people mainly use Reddit for its entertainment value (Bogers \& Wernersen, 2014).

Banter serves to facilitate social interactions among group members while simultaneously excluding people who do not understand or enjoy the banter. Online, banter and other "offtopic" messages explicate common group bonds; in fact, one researcher suggests that affiliation through laughter and humor "accounts for communal identification of [users in online discussion groups] into communities of bonds" (Knight, 2010, p. 35). Therefore, users in online communities may banter and joke as a way to test and explicate affiliation, cohesion, and similarity among users. Similarity assessment has been found to be a central component of information behavior (e.g., seeking and disclosure) in other online contexts, most notably in online support groups for health (Costello, 2015). Therefore, on these subreddits, banter functions as a type of similarity or homophily assessment, an assessment of relevance based on social cues that fosters group cohesion and creates a social world. In this particular instance, it facilitates disclosure of incriminating behaviors in both subreddits.

Banter on these subreddits also demonstrates that users likely visit these online spaces not to primarily search for or disclose information, but to engage in social interaction with likeminded individuals. Therefore, Reddit serves as an information ground, where multiple different sub-contexts operate together and form a "grand context" for information transfer among people (Fisher, Landry, \& Naumer, 2007). With a higher prevalence of banter, $r /$ trees may be more readily classified as an information ground; this is also corroborated by the number of users in $r$ /trees (875) compared with the number of users in $r$ /opiates (355). The smaller number of users in $\mathrm{r}$ /opiates allows for individual users to more readily recognize one another and to determine the credibility of specific users over time.

Disclosure on both subreddits is also shaped by norms on each subreddit. The discussions in r/opiates contain more information seeking and information sharing, as people both ask for and share specific instructional advice about use and addiction. These comments are not as common in $\mathrm{r} /$ trees. This reflects both the cultural attitudes about these substances-marijuana is less stigmatized in comparison with heroin (Brown, 2015) — as well as the practical realities of the drugs: marijuana use is less dangerous than opiate use; therefore, people may have less of a need for advice about how to use marijuana from other users. In many cases, disclosure is necessary in order to provide sufficient context for questions about safely consuming opiates. It is also used to lend credibility to answers, particularly in the r/opiates subreddit. This study therefore supports the claim that information seeking and disclosure are often linked processes in online interactions (Costello, 2015). 
In contrast with the discussions on $\mathrm{r}$ /opiates, many people in $\mathrm{r}$ /trees discuss the medicinal uses of marijuana, disclosing their use in the process. This is a surprising contrast, because pain medications are a frequent topic of discussion in r/opiates, although the commenters there do not often discuss using opioids to manage chronic pain. Another striking difference between the types of disclosure in these subreddits is that opiate users tend to ask information about how to administer drugs, while these conversations are rare on $\mathrm{r} /$ trees. As such, there is much less advice on $\mathrm{r}$ /trees. This may be because the repercussions of ingesting marijuana incorrectly are much less serious: opiates are much more dangerous. Furthermore, conversations about overdoses are common in r/opiates, in contrast with $\mathrm{r} /$ trees, where overdoses are not discussed. In fact 18,893 people in the United States died from prescription opiate overdose in 2014, and another 10,754 people died from heroin in the same year (National Institute on Drug Abuse, 2015). While there are health risks associated with marijuana use, the public perception is that there are none (Hughes, Lipari, \& Williams, 2015). This is reflected in the type of discussions people have on each subreddit: $r$ /trees has much more banter and much less instruction, while r/opiates has a larger amount of instruction than would expected in our sample.. More research is necessary to determine the motivations for use of online forums focused on illicit or illegal behaviors; a large-scale survey and interviews with select participants in these spaces is a potential next step for this work.

\section{Conclusion}

In this article, we set out to examine how people discuss incriminating or illicit behavior online. We focused on two subreddits for online discussions about illicit drug use: r/opiates and $\mathrm{r} /$ trees, and found that people disclose drug use fairly frequently on both subreddits, although there is also a fair amount of off-topic banter. People also give instructional advice, talk about drug culture, moralize, and discuss community norms on both subreddits. There are two significant differences between the subreddits: there is more banter in $r$ /trees than in $r$ /opiates, and there is more instruction in $\mathrm{r} /$ opiates than in $\mathrm{r} /$ trees. These differences can largely be attributed to the type of needs that people have when they visit the forums: in r/opiates, people are looking for credible information about how to safely ingest opioid drugs, while this is not a need that people have when ingesting marijuana. We also found that disclosure of drug use often occurs because people have questions about drug use, or because they want to lend credibility to the personal experiences they're sharing online. In this way, disclosure serves as a crucial information behavior in these discussions. This study therefore fills gaps in our understanding of the interplay between information seeking and personal information disclosure. In this context, disclosure operates to strengthen credibility of information and advice as it is shared.

It is possible that our results are specific to the two subreddits we examined in this study. However, this is an exploratory study and the results are not intended to be generalizable. Instead, we are focused on the transferability of our findings to other, similar contexts. An important contribution we make with this study is the development of a codebook for online disclosure of illicit behaviors. Future work could apply the codebook deductively to online disclosure about other illicit and stigmatized activities in order to determine whether these findings are transferrable to other contexts. It may also be possible to automatically detect instances of disclosure of illicit behaviors online, a possibility to be explored with further study. Finally, public health interventions could be developed that harness our findings. For example, embedding public health officials within existing online drug forums and encouraging them to 
engage in banter may be a useful tactic for sharing credible information about illicit and potentially dangerous substances like heroin with users who are seeking drug information online.

\section{References}

Agresti, A., \& Kateri, M. (2011). Categorical Data Analysis. In M. Lovric (Ed.), International Encyclopedia of Statistical Science (pp. 206-208). Springer Berlin Heidelberg. https://doi.org/10.1007/978-3-642-04898-2_161

Ahern, J., Stuber, J., \& Galea, S. (2007). Stigma, discrimination and the health of illicit drug users. Drug and Alcohol Dependence, 88(2-3), 188-196. https://doi.org/10.1016/j.drugalcdep.2006.10.014

Balani, S., \& De Choudhury, M. (2015). Detecting and Characterizing Mental Health Related SelfDisclosure in Social Media. In Proceedings of the 33rd Annual ACM Conference Extended Abstracts on Human Factors in Computing Systems (pp. 1373-1378). New York, NY, USA: ACM. https://doi.org/10.1145/2702613.2732733

Bancroft, A., \& Reid, P. S. (2015). Concepts of illicit drug quality among darknet market users: Purity, embodied experience, craft and chemical knowledge. International Journal of Drug Policy. https://doi.org/10.1016/j.drugpo.2015.11.008

Barratt, M. J. (2011). Discussing Illicit Drugs in Public Internet Forums: Visibility, Stigma, and Pseudonymity. In Proceedings of the 5th International Conference on Communities and Technologies (pp. 159-168). New York, NY, USA: ACM.

https://doi.org/10.1145/2103354.2103376

Barratt, M. J., Lenton, S., Maddox, A., \& Allen, M. (2016). "What if you live on top of a bakery and you like cakes?"-Drug use and harm trajectories before, during and after the emergence of Silk Road. International Journal of Drug Policy. https://doi.org/10.1016/j.drugpo.2016.04.006

Becker, H. S. (1953). Becoming a marihuana user. American Journal of Sociology, 59(3), 235-242. Bergman, M. M. (2010). Hermeneutic Content Analysis: Textual and Audiovisual Analyses within a Mixed Methods Framework. In A. Tashakkori \& C. Teddlie, SAGE Handbook of Mixed 
Methods in Social \& Behavioral Research (pp. 379-396). 2455 Teller Road, Thousand Oaks California 91320 United States: SAGE Publications, Inc. Retrieved from http://methods.sagepub.com/book/sage-handbook-of-mixed-methods-socialbehavioral-research-2e/n16.xml

Bogers, T., \& Wernersen, R. (2014). How social are social news sites? Exploring the motivations for using Reddit. com. In iConference 2014 Proceedings. Berlin, Germany. Retrieved from https://www.ideals.illinois.edu/handle/2142/47295

Bowles, H., \& Moretti, V. (2016). Negotiating legal identity online: Narratives of drug use. Language in the Negotiation of Justice: Contexts, Issues and Applications, 167.

Brown, S. A. (2015). Stigma towards Marijuana Users and Heroin Users. Journal of Psychoactive Drugs, 47(3), 213-220. https://doi.org/10.1080/02791072.2015.1056891

Case, D. O. (2012). Looking for Information: A Survey of Research on Information Seeking, Needs and Behavior (3rd ed.). Emerald Group Publishing.

Costello, K. L. (2015). Social information behaviors in the context of chronic kidney disease: Information seeking and disclosure in online support groups (Doctoral dissertation). University of North Carolina, Chapel Hill, NC.

File, C., \& Bahney, B. (2012). Characterizing incriminating information sharing in online communities using strategic SIDE. Lawrence Livermore National Laboratory. Retrieved from https://cyberdefender.llnl.gov/content/assets/docs/Files_August2012.pdf

Fisher, K. E., Landry, C. F., \& Naumer, C. (2007). Social spaces, casual interactions, meaningful exchanges:'information ground'characteristics based on the college student experience. Information Research, 12(2). Retrieved from http://www.informationr.net/ir/122/paper291.html

Hughes, A., Lipari, R. N., \& Williams, M. (2015). State estimates of adolescent marijuana use and perceptions of risk of harm from marijuana use: 2013 and 2014 (Short report). Rockville, MD: Substance Abuse; Mental Health Services Administration. Retrieved from http://www.samhsa.gov/data/sites/default/files/report_2121/ShortReport-2121.pdf 
Isaac, M. (2014, September 8). Reddit and 4chan begin to button up. Retrieved from http://bits.blogs.nytimes.com/2014/09/08/reddit-and-4chan-begin-to-button-up/.

Knight, N. K. (2010). Wrinkling complexity: Concepts of identity and affiliation in humor. In M. Bednarek \& J. R. Martin (Eds.), New discourse on language: Functional perspectives on multimodality, identity, and affiliation (pp. 35-58). London: Continuum.

Månsson, J., \& Ekendahl, M. (2013). Legitimacy through scaremongering: The discursive role of alcohol in online discussions of cannabis use and policy. Addiction Research \& Theory, 21(6), 469-478. https://doi.org/10.3109/16066359.2012.731115

Massanari, A. (2015). \#Gamergate and The Fappening: How Reddit's algorithm, governance, and culture support toxic technocultures. New Media \& Society, 1461444815608807. https://doi.org/10.1177/1461444815608807

Murguia, E., Tackett-Gibson, M., \& Lessem, A. (2007). Real Drugs in a Virtual World: Drug Discourse and Community Online. Lexington Books.

Norris, N. (1997). Error, bias and validity in qualitative research. Educational Action Research, 5(1), 172-176. https://doi.org/10.1080/09650799700200020

O’Brien, K., Chatwin, C., Jenkins, C., \& Measham, F. (2015). New psychoactive substances and British drug policy: A view from the cyber-psychonauts. Drugs: Education, Prevention and Policy, 22(3), 217-223. https://doi.org/10.3109/09687637.2014.989959

Peddinti, S. T., Ross, K. W., \& Cappos, J. (2014). “On the Internet, Nobody Knows You’Re a Dog”: A Twitter Case Study of Anonymity in Social Networks. In Proceedings of the Second ACM Conference on Online Social Networks (pp. 83-94). New York, NY, USA: ACM. https://doi.org/10.1145/2660460.2660467

Pedersen, D. M. (1997). Psychological functions of privacy. Journal of Environmental Psychology, 17(2), 147-156.

Petronio, S. S. (2002). Boundaries of privacy: Dialectics of disclosure. Albany, NY: SUNY Press. Plester, B. A., \& Sayers, J. (2007). “Taking the piss”: Functions of banter in the IT industry. Humor: International Journal of Humor Research, 20(2), 157-187. 
Saldana, J. (2015). The Coding Manual for Qualitative Researchers (3rd edition). Los Angeles, Calif. ; London: SAGE Publications Ltd.

Substance Abuse and Mental Health Services Administration. (2015). Behavioral Health Barometer: United States, 2015 (No. HHS Publication No. SMA-16-Baro-2015). Rockville, MD: Substance Abuse and Mental Health Services Administration.

Tackett-Gibson, M. (2008). Constructions of Risk and Harm in Online Discussions of Ketamine Use. Addiction Research \& Theory, 16(3), 245-257. https://doi.org/10.1080/16066350801983699

Thomas, J. O., Dunn, M., Swift, W., \& Burns, L. (2011). Illicit drug knowledge and informationseeking behaviours among elite athletes. Journal of Science and Medicine in Sport, 14(4), 278-282. https://doi.org/10.1016/j.jsams.2011.02.001

Todd, R. J. (1999). Utilization of heroin information by adolescent girls in Australia: A cognitive analysis. Journal of the American Society for Information Science, 50(1), 10-23. https://doi.org/10.1002/(SICI)1097-4571(1999)50:1<10::AID-ASI4>3.0.CO;2-B

Wakefield, M. A., Loken, B., \& Hornik, R. C. (2010). Use of mass media campaigns to change health behaviour. The Lancet, 376(9748), 1261-1271. https://doi.org/10.1016/S0140$6736(10) 60809-4$

Žegarac, V. (1998). What is“ Phatic Communication”? In Current issues in relevance theory (pp. 327-362). University College London: John Benjamins Publishing Company. Retrieved from dx.doi.org/10.1075/pbns.58

Zheluk, A., Quinn, C., \& Meylakhs, P. (2014). Internet Search and Krokodil in the Russian Federation: An Infoveillance Study. Journal of Medical Internet Research, 16(9). https://doi.org/10.2196/jmir.3203 